\title{
Influence of foetal inflammation on the development of meconium aspiration syndrome in term neonates with meconium-stained amniotic fluid
}

\author{
Kyoko Yokoi $^{\text {Corresp., }}{ }^{1}$, Osuke Iwata ${ }^{2}$, Satoru Kobayashi ${ }^{1}$, Kanji Muramatsu ${ }^{1}$, Haruo Goto ${ }^{1}$ \\ ${ }^{1}$ Department of Pediatrics, Nagoya West Medical Center, Nagoya, Japan \\ Department of Neonatology and Pediatrics, Nagoya City University Graduate School of Medical Science, Nagoya, Japan \\ Corresponding Author: Kyoko Yokoi \\ Email address: kyoyo0410@yahoo.co.jp
}

Background: Meconium-stained amniotic fluid is observed in approximately $10-15 \%$ of all deliveries, however, only $5 \%$ of neonates with meconium-stained amniotic fluid develop meconium aspiration syndrome (MAS). Although foetal distress and subsequent sympathetic stimulation have been considered as the primary upstream events of MAS, this clinical complication sometimes occurs due to other pathologies, such as intraamniotic inflammation. The aim of this study was to investigate whether the incidence of MAS is associated with the presence of funisitis and chorioamnionitis in term neonates with meconium-stained amniotic fluid.

Methods: Between April 2013 and March 2015, 95 term neonates with meconium-stained amniotic fluid, who were hospitalized at a neonatal intensive care unit, were enrolled in the study. The placenta and umbilical cord were histopathologically examined. Clinical variables and histopathological findings associated with the incidence of MAS were studied.

Results: A total of 36 neonates developed MAS. Univariate logistic regression analysis revealed that a heavier birth weight, male sex, 1-min Apgar score $\leq 7$, funisitis (but not chorioamnionitis), and elevated acute-phase inflammatory reaction score were associated with increased incidence of MAS $(a l l p<0.05)$. The multivariate model comprised funisitis $(\mathrm{OR}=5.03,95 \% \mathrm{Cl}, 1.63-15.5)$, 1-min Apgar score $\leq 7$ $(\mathrm{OR}=2.74,95 \% \mathrm{Cl}, 1.06-7.09)$, and male sex $(\mathrm{OR}=3.4,95 \% \mathrm{Cl}, 1.24-9.34)$.

Conclusion: In neonates with meconium-stained amniotic fluid, funisitis, as well as low 1-min Apgar score and male sex, was identified as an independent variable for MAS development. Intraamniotic inflammation might be involved in the pathological mechanisms of MAS. 
1 Influence of Foetal Inflammation on the Development of Meconium

2 Aspiration Syndrome in Term Neonates with Meconium-Stained

3 Amniotic Fluid

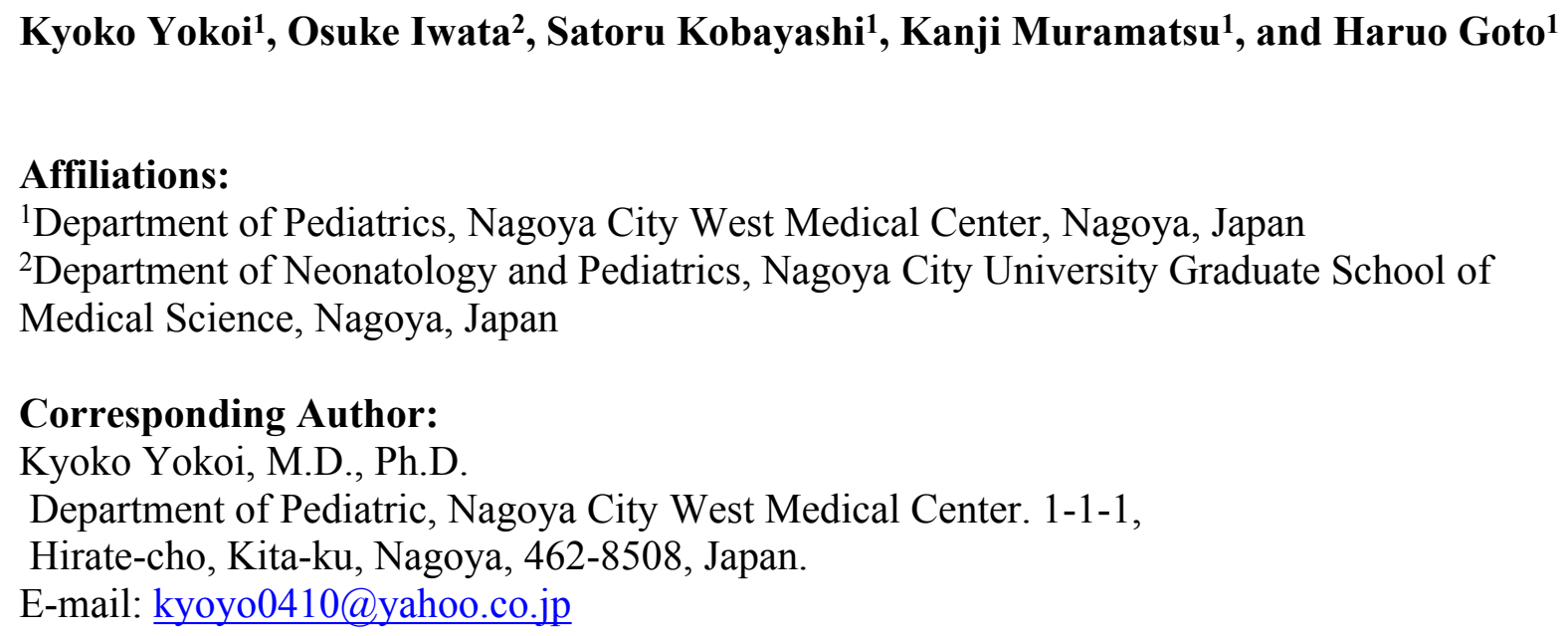


45 Abstract

46

47 Background: Meconium-stained amniotic fluid is observed in approximately $10-15 \%$ of all

48 deliveries, however, only $5 \%$ of neonates with meconium-stained amniotic fluid develop

49 meconium aspiration syndrome (MAS). Although foetal distress and subsequent sympathetic

50 stimulation have been considered as the primary upstream events of MAS, this clinical

51 complication sometimes occurs due to other pathologies, such as intraamniotic inflammation.

52 The aim of this study was to investigate whether the incidence of MAS is associated with the

53 presence of funisitis and chorioamnionitis in term neonates with meconium-stained amniotic

54 fluid.

55 Methods: Between April 2013 and March 2015, 95 term neonates with meconium-stained

56 amniotic fluid, who were hospitalized at a neonatal intensive care unit, were enrolled in the

57 study. The placenta and umbilical cord were histopathologically examined. Clinical variables

58 and histopathological findings associated with the incidence of MAS were studied.

59 Results: A total of 36 neonates developed MAS. Univariate logistic regression analysis revealed

60 that a heavier birth weight, male sex, 1-min Apgar score $\leq 7$, funisitis (but not chorioamnionitis),

61 and elevated acute-phase inflammatory reaction score were associated with increased incidence

62 of MAS (all $\mathrm{p}<0.05)$. The multivariate model comprised funisitis $(\mathrm{OR}=5.03,95 \% \mathrm{CI}, 1.63-15.5)$,

63 1-min Apgar score $\leq 7(\mathrm{OR}=2.74,95 \% \mathrm{CI}, 1.06-7.09)$, and male sex (OR=3.4, 95\%CI, 1.24-9.34).

64 Conclusion: In neonates with meconium-stained amniotic fluid, funisitis, as well as low 1-min

65 Apgar score and male sex, was identified as an independent variable for MAS development.

66 Intraamniotic inflammation might be involved in the pathological mechanisms of MAS.

67

68

69

70
Introduction

Peer] reviewing PDF | (2018:12:33771:1:1:NEW 22 Mar 2019) 
71 Meconium-stained amniotic fluid is observed in approximately 10-15\% of all deliveries (Yoder 72 et al., 2002), which has been considered as the primary cause of meconium aspiration syndrome

73 (MAS). However, only 5\% of neonates with meconium-stained amniotic fluid develop MAS

74 (Yoder et al., 2002; Dargaville et al., 2006; Swarnam et al., 2012). In addition, the use of

75 surfactant replacement, nitric oxide therapy and extracorporeal membrane oxygenation has led to

76 a marked decrease in mortality and morbidity associated with MAS in the recent times (Swarnam

77 et al. 2012). Nevertheless, these therapeutic options are usually unavailable for neonates born in

78 a low-resource setting. Even in a high-resource setting, advanced intervention is often delayed,

79 because the current diagnostic algorithms of MAS based on clinical symptoms and X-ray

80 patterns do not provide sufficient insight as to who are likely to develop severe respiratory

81 failure (Ahanya et al., 2005; Swarnam et al., 2012).

82 To establish early, reliable biomarkers of MAS, precise understanding of the pathological

83 mechanism is essential. Although asphyxia and subsequent sympathetic stimulation have been

84 considered to trigger foetal passage of meconium into amniotic fluid (Ahanya et al., 2005; van

85 Ierland et al., 2009; Swarnam et al., 2012; Lindenskov et al., 2015), MAS sometimes develops in

86 the absence of overt episodes suggestive of antenatal asphyxia; several previous studies did not

87 find an association between low Apgar scores, umbilical arterial acidosis, and incidence of MAS

88 (Trimmer et al., 1991; Ramin et al., 1993; Blackwell et al., 2001; Oyelese et al., 2006),

89 suggesting the presence of additional triggers of MAS, such as intraamniotic inflammation.

90 Studies suggested that meconium-stained amniotic fluid is more likely to be contaminated with

91 bacteria, endotoxin, and inflammatory mediators than clear amniotic fluid, and often

92 accompanied by clinical evidence of intraamniotic inflammation (Romero et al., 2014).

93 Meconium-stained amniotic fluid is also linked to findings suggestive of intraamniotic 
94 inflammation, which is represented by funisitis and chorioamnionitis (Burgess et al., 1996;

95 Romero et al., 2014; Choi et al., 2015; Lee et al., 2016). While chorioamnionitis is suggestive of

96 maternal inflammatory response, funisitis is considered to represent foetal inflammatory

97 response (Redline et al., 2003). In preterm neonates with funisitis, elevated plasma interleukin-6

98 (IL-6) level in cord blood (Yoon et al., 2000) is associated with increased incidence of adverse

99 neonatal outcomes such as chronic lung disease, intracranial haemorrhage, and cerebral palsy

100 (Kim et al., 2001; Yoon et al., 2000). However, few studies in term neonates have investigated

101 the influence of intraamniotic inflammation to the development of MAS.

102 We performed a prospective observational study to investigate whether the incidence of MAS is 103 associated with the presence of funisitis and chorioamnionitis.

104

105 Methods

106 Patients and Methods

107 This study was conducted under the approval of the Ethics Committee of Nagoya West Medical 108 109 provides intensive care for virtually all sick neonates, except for those who require advanced life 116 support treatments, such as haemodialysis/filtration, cardiac surgery, and extracorporeal 117 membrane oxygenation. Of these, 239 inborn neonates $\geq 37$ weeks of gestation were admitted 
118 within $24 \mathrm{~h}$ of birth, whose blood samples on day 0 were available. Subsequently, 99 neonates

119 were enrolled in the study after excluding 10 neonates with major congenital anomalies and 130

120 neonates without meconium-stained amniotic fluid (Figure 1).

121

122 Clinical variables

123 Presence of meconium-stained amniotic fluid was evaluated at birth under visual observation as 124 previously described (Lee et al., 2016). MAS was defined as respiratory distress in neonates 125 though meconium-stained amniotic fluid whose symptoms cannot be otherwise explained, 126 requiring assisted mechanical ventilation or oxygen at a concentration of $\geq 40 \%$ for at least $48 \mathrm{~h}$, 127 and radiographic findings were consistent with MAS (Fraser et al., 2005; Lee et al., 2016). X-ray 128 photographs were reviewed by an experienced staff neonatologist, and the placenta and umbilical 129 cord were histopathologically examined by a single pathologist. Chorioamnionitis was defined as 130 infiltration of neutrophils identified in chorionic membranes (Stage II by Blanc's classification), 131 whereas funisitis was defined as infiltration of neutrophils within the walls of umbilical vessels 132 or in Wharton's jelly (Blanc et al., 1979; Redline et al., 2003). Other clinical variables were 133 collected from patients' clinical record, including gestational age, birth weight and its standard 134 score calculated against the standard Japanese birth weight for parity and gestational age 135 (Itabashi K et al. 2010), sex, delivery mode, premature rupture of the membranes, Apgar score at 1361 and $5 \mathrm{~min}$, cord blood $\mathrm{pH}$, and the duration of invasive/non-invasive positive-pressure 137 ventilation and oxygen supplementation. 
140 In our unit, at the admission of neonates born through meconium-stained amniotic fluid, routine

141 blood tests are performed including blood gas analysis, cell counts, blood sugar level, and serum

142 inflammatory markers. For the inflammatory markers, C-reactive protein (CRP), $\alpha_{1}$-acid

143 glycoprotein $\left(\alpha_{1} A G\right)$, and haptoglobin were measured using turbidimetric immunoassay (Quick

144 Turbo, Shino-Test Corporation, Japan). CRP $>0.3 \mathrm{mg} / \mathrm{dL}, \alpha_{1} \mathrm{AG}>20 \mathrm{mg} / \mathrm{dL}$, and haptoglobin

$145>13 \mathrm{mg} / \mathrm{dl}$ were regarded as positive (Speer et al., 1983; Ipek et al., 2010; Nakamura et al.,

146 2015); the acute-phase inflammatory reaction scores of 0 (none positive), 1 (one positive

147 biomarker), 2 (two positive biomarkers) and 3 (all three positive biomarkers) were given based

148 on the number of positive inflammatory biomarkers (Nakamura et al., 2015). Venous blood

149 culture was assessed for neonates with clinical signs suggestive of antenatal infection (e.g.

150 elevation of maternal white blood cell count and CRP, maternal pyrexia, and premature rupture

151 of the membranes) or with the acute-phase inflammatory reaction scores $\geq 1$.

152

153 Data analysis

154 The clinical variables were compared between neonates with and without MAS. Apgar scores $155(\leq 7)$ and cord blood $\mathrm{pH}(<7.1)$ were divided using clinically relevant thresholds. All statistical 156 analyses were performed using R (The R Foundation for Statistical Computing, Vienna, Austria, 157 version 1.22) and its Japanese interface (EZR; Saitama Medical Center, Jichi Medical

158 University, Saitama, Japan) (Kanda, 2013). Logistic regression analyses were performed to 159 develop a model explaining MAS development using funisitis and chorioamnionitis as 160 mandatory independent variables (only one of the two mandatory variables were tested at a time 161 because of the collinearity observed between each other). Statistical findings were not corrected 162 for multiple comparisons for univariate analysis, because of the exploratory nature of this study. 
164 Results

165 Of 99 term neonates with meconium-stained amniotic fluid, histopathological examination 166 was not available in 4 neonates, whose data were not considered further. Of 95 remaining 167 neonates, 36 neonates $(37.9 \%)$ developed MAS, none of whom showed positive blood culture. 168 Gestational age, caesarean delivery, and incidence of premature rupture of the membranes and 169 cord blood acidosis did not differ between neonates who developed and did not develop MAS 170 (Table 1). Univariate logistic regression analysis showed that a high birth weight, male sex, 1171 min Apgar score $\leq 7$, funisitis, and a high acute-phase inflammatory reaction score of 3

172 (compared with 0) were associated with increased incidence of MAS (all p<0.05; Table 1). The 173 final model to explain the incidence of MAS in neonates with meconium-stained amniotic fluid 174 comprised funisitis (OR, 5.03; 95\%CL, 1.63-15.5), low 1-minute Apgar score $\leq 7$ (OR, 2.74; 175 95\%CL, 1.06-7.09), and male sex (OR, 3.4; 95\%CL, 1.24- 9.34), where chorioamnionitis was 176 not identified as an independent variable to explain MAS development (See Supplemental Table 1771 for the multivariate model, which used chorioamnionitis instead of funisitis; and Supplemental 178 Tables 2 and 3 for the multivariate model, which used the duration of mechanical ventilation and 179 oxygen supplementation as alternative dependent variables).

180

181

\section{Discussion}

MAS is one of the most commonly recognized respiratory diseases in neonates, however, the exact pathological mechanism leading to foetal meconium passage into the amniotic fluid and its aspiration is still unknown. Our study suggested that, in neonates with meconium-stained amniotic fluid, funisitis, as well as low 1-min Apgar scores and male sex, was associated with increased incidence of MAS, pointing towards the role of inflammatory factors in the 
187 pathophysiology of MAS. Further large-scale studies are required to delineate the role of 188 antenatal inflammation in the development of meconium-stained amniotic fluid and MAS.

189 Previous studies highlighted that MAS development is associated with low cord blood $\mathrm{pH}$ and 190 Apgar scores at 5 min (Dargaville et al., 2006; van lerland et al., 2009; Karabayir et al., 2015), 191 supporting the hypothesis that hypoxic-ischemic events trigger foetal meconium passage into the 192 amniotic fluid and its subsequent aspiration. However, several studies failed to demonstrate the 193 association between cord blood pH, Apgar scores and MAS development (Trimmer et al., 1991; 194 Ramin et al., 1993; Blackwell et al., 2001; Oyelese et al., 2006). Our study also found that only 195 the 1-min Apgar scores, but not the 5-min Apgar scores and cord blood pH, were associated with 196 MAS development, suggesting that antenatal hypoxic-ischemia is not sufficient to explain MAS 197 development, and other control variables of MAS might exist.

198 In our study, funisitis was associated with an increased incidence of MAS, supporting a 199 hypothesis that intraamniotic inflammation might be an additional factor involved in the 200 development of MAS. A study that investigated autopsy cases with histopathologic evidence of 201 intrauterine meconium exposure found that pulmonary inflammation was associated with 202 inflammation of the umbilical cord, as well as meconium aspiration (Romero et al., 2014).

203 Thureen et al. showed that three of six placentas with MAS showed funisitis associated with 204 villus ischemic changes, which may have contributed to in utero foetal hypoxia (Thureen et al., 205 1997). Lee et al. showed that neonates diagnosed with funisitis were at more than 4-fold risks of 206 developing MAS than those without funisitis, whereas Kim et al. observed that the incidence and 207 severity of funisitis were associated with those of MAS (Lee et al., 2016; Kim et al., 2017), 208 suggesting that intraamniotic inflammation with foetal systemic inflammation might be an 209 important upstream control variable in MAS (Lee et al., 2016). By performing placental 
210 histopathological examination virtually for all NICU neonates, our study provided additional

211 evidence to support the impact of intraamniotic inflammation to MAS development.

212 In our study, MAS development was associated with funisitis, but not chorioamnionitis. It is

213 possible that chorioamnionitis might represent a maternal inflammatory response to

214 intraamniotic infection, whereas funisitis might be predominantly represent the foetal

215 inflammatory response, which is accompanied by elevation of inflammatory cytokine and

216 procytokine levels. Intraamniotic inflammation occurs more frequently in the presence of

217 meconium-stained amniotic fluid than that in clear amniotic fluid, as shown by elevated amniotic

218 fluid IL-6 levels in neonates with meconium-stained amniotic fluid (Romero et al., 2014). In

219 animal models, foetal swallowing of amniotic fluid containing bacteria, endotoxin and

220 proinflammatory mediator is suggested to evoke inflammation and subsequent bowel peristalsis

221 and meconium passage, which may explain both meconium passage in utero and its aspiration in

222 association with foetal infection and inflammation. A clinical study confirmed that meconium

223 with bacteria and inflammatory mediators may be aspirated in utero following stressful events

224 with or without acidaemia. It is possible to extrapolate that intraamniotic inflammation triggers a

225 range of foetal inflammatory response, which is represented by funisitis and elevation of

226 inflammatory biomarkers. Foetal ingestion of amniotic fluid containing inflammatory mediators

227 may ultimately induce the meconium passage into the amniotic fluid. Although our study did not

228 measure inflammatory cytokine levels, three inflammatory biomarkers, CRP, $\alpha_{1} \mathrm{AG}$, and

229 haptoglobin, were employed, which are produced in the liver in response to intrinsic/extrinsic

230 stimuli and subsequent elevation of IL-1ß and IL-6 (Ipek et al., 2010; Nakamura et al., 2015).

231 Although these inflammatory biomarkers and their composite scores were not involved within

232 the final model to explain the development of MAS, there was a consistent trend that an increase 
233 in inflammatory biomarkers was associated with relatively higher incidence of MAS. Consistent

234 to our findings, Hofer et al. reported that elevated CRP levels and changes in white blood

235 cell/neutrophil counts were associated with the severity of MAS, although this study

236 simultaneously highlighted the potential impact of mechanical ventilation on the inflammatory

237 biomarkers (Hofer et al., 2016). Further accumulation of knowledge regarding the role of

238 inflammatory reactions may accelerate the establishment of biomarkers, which provide early,

239 precise prediction of MAS development in neonates, who are born through meconium-stained

240 amniotic fluid.

241 It is known that inflammatory responses are sex-dependent; for example, studies in vivo

242 demonstrated greater release of proinflammatory cytokines after in vivo lipopolysaccharide

243 stimulation in male foetuses than in female foetuses (Kim-Fine et al., 2012; Koch et al., 2014).

244 Lambermont and colleagues reported increased white blood count response in bronchoalveolar

245 fluid and worse lung compliance following exposure to chorioamnionitis in male than in female

246 neonates (Lambermont et al., 2012). In our study, male neonates had at a higher risk of

247 developing MAS than female neonates. However, the size of our study population was too small

248 to confirm the role of sex on MAS development.

249 Several limitations of our study were noted, which need to be considered when interpreting

250 our findings. Although we performed standard histopathological examinations of the placenta

251 and umbilical cord for all participating neonates, the study population is relatively small, which

252 did not allow the assessment of some important clinical cofounders. To minimize the

253 population bias, our study was conducted at a single level-II unit recruiting only inborn

254 neonates. However, we were not able to include neonates, who did not have meconium-stained

255 amniotic fluid, and those, who were not hospitalized at NICU, leaving uncertainly around the 
256 role of intraamniotic inflammation in the entire birth cohort. Several important clinical variables

257 associated with antenatal inflammation and hypoxic-ischemic stress, such as maternal fever,

258 infection and finding from cardiotocography, were not incorporated within the analysis, leading

259 to a difficulty in discriminating acute chorioamnionitis and funisitis from chronic ones. A

260 prospective cohort study is currently underway to elucidate the role of intraamniotic

261 inflammation on MAS development in all neonates born at our hospital by involving extensive

262 maternal and neonatal variables.

263

264 Conclusions

265

266

267

268

mechanisms of MAS. Further large-scale prospective studies are needed to delineate the detailed

269 mechanism of MAS and allow its efficient prediction and prevention.

270

271

272

273

274

275

276

277

278

279

280

281 282

Acknowledgements

The authors are grateful to all clinical staffs of the Division of Obstetrics and Gynecology and the Division of Clinical Pathology, Nagoya West Medical Center, for their technical input in the histopathological examination of the placenta.

\section{References}

Ahanya SN, Lakshmanan J, Morgan BL, and Ross MG. 2005. Meconium passage inutero: mechanisms, consequences, and management. Obstet Gynecol Surv 60:45-56; uiz 73-44. 
283 Blackwell SC, Moldenhauer J, Hassan SS, Redman ME, Refuerzo JS, Berry SM, and

284 Sorokin Y. 2001. Meconium aspiration syndrome in term neonates with normal acid-base

285 status at delivery: is it different? Am J Obstet Gynecol 184:1422-1425; discussion 14252861426.

287 Blanc WA. 1979. Pathology of the placenta and cord in ascending and in haematogenous 288 infection. Ciba Found Symp:17-38.

289 Burgess AM, and Hutchins GM. 1996. Inflammation of the lungs, umbilical cord and 290 placenta associated with meconium passage in utero. Review of 123 autopsied cases. 291 Pathol Res Pract 192:1121-1128 DOI: 10.1016/S0344-0338(96)80029-X

292 Choi W, Jeong H, Choi SJ, Oh SY, Kim JS, Roh CR, and Kim JH. 2015. Risk factors 293 differentiating mild/moderate from severe meconium aspiration syndrome in meconium294 stained neonates. Obstet Gynecol Sci 58:24-31 DOI: 10.5468/ogs.2015.58.1.24

295 Dargaville PA, Copnell B, Australian, and New Zealand Neonatal N. 2006. The 296 epidemiology of meconium aspiration syndrome: incidence, risk factors, therapies, and 297 outcome. Pediatrics 117:1712-1721. DOI: 10.1542/peds.2005-2215Fraser WD, Hofmeyr J, Lede 298 R, Faron G, Alexander S, Goffinet F, Ohlsson A, Goulet C, 299 Turcot-Lemay L, Prendiville W, Marcoux S, Laperriere L, Roy C, Petrou S, Xu HR, Wei 300 B, and Amnioinfusion Trial G. 2005. Amnioinfusion for the prevention of the meconium 301 aspiration syndrome. $N$ Engl J Med 353:909-917 DOI: 10.1056/NEJMoa050223

302 Hofer N, Jank K, Strenger V, Pansy J, and Resch B. 2016. Inflammatory indices in 303 meconiumaspiration syndrome. Pediatr Pulmonol 51: 601-606 DOI: 10.11002/ppul.23349

304 Ipek IO, Saracoglu M, and Bozaykut A. 2010. Alpha1-acid glycoprotein for the early 305 diagnosis of neonatal sepsis. J Matern Fetal Neonatal Med 23:617-621 DOI: 
306

307

308

309

310

311

312

313

314

315

316

317

318

319

320

321

322

323

324

325

326

327

328

$10.1080 / 14767050903258720$

Itabashi K, Miura F, Uehara R, Nakamura K. 2014. New Japanese neonatal anthropometric chart for gestational age at birth. Pediatr Int 56:702-708 DOI: 10.1111/ped.12331

Kanda Y. 2013. Investigation of the freely available easy-to-use software 'EZR' for medical statistics. Bone Marrow Transplant 48:452-458 DOI: 10.1038/bmt.2012.244

Karabayir N, Demirel A, and Bayramoglu E. 2015. Blood lactate level and meconium aspiration syndrome. Arch Gynecol Obstet 291:849-853 DOI: 10.1007/s00404-014-3482-3

Kim B, Oh SY, and Kim JS. 2017. Placental Lesions in Meconium Aspiration Syndrome.

J Pathol Transl Med 51:488-498 DOI: 10.4132/jptm.2017.07.20

Kim CJ, Yoon BH, Romero R, Moon JB, Kim M, Park SS, and Chi JG. 2001. Umbilical arteritis and phlebitis mark different stages of the fetal inflammatory response. Am J

Obstet Gynecol 185:496-500 DOI: 10.1067/mob.2001.116689

Kim-Fine S, Regnault TR, Lee JS, Gimbel SA, Greenspoon JA, Fairbairn J, Summers K, and de Vrijer B. 2012. Male gender promotes an increased inflammatory response to lipopolysaccharide in umbilical vein blood. J Matern Fetal Neonatal Med 25:2470-2474 DOI:

$10.3109 / 14767058.2012 .684165$

Koch FR, Wagner CL, Jenkins DD, Caplan MJ, Perkel JK, Rollins LG, Katikaneni LD, and Mulvihill DM. 2014. Sex differences in cerebral blood flow following chorioamnionitis in healthy term infants. J Perinatol 34:197-202 DOI: 10.1038/jp.2013.179 Lambermont VA, Been JV, Kunzmann S, Vanterpool SF, Newnham JP, Kallapur SG, Jobe AH, and Kramer BW. 2012. Sex differences in lung gas volumes after lipopolysaccharide-induced chorioamnionitis in fetal sheep. Gend Med 9:278-286 DOI: 10.1016/j.genm.2012.05.005 
329 Lee J, Romero R, Lee KA, Kim EN, Korzeniewski SJ, Chaemsaithong P, and Yoon BH.

330 2016. Meconium aspiration syndrome: a role for fetal systemic inflammation. Am J

331 Obstet Gynecol 214:366 e361-369 DOI: 10.1016/j.ajog.2015.10.009

332 Lindenskov PH, Castellheim A, Saugstad OD, and Mollnes TE. 2015. Meconium

333 aspiration syndrome: possible pathophysiological mechanisms and future potential

334 therapies. Neonatology 107:225-230 DOI: 10.1159/000369373

335 Nakamura T, Hatanaka D, Yoshioka T, Yamada S, and Goto H. 2015. Study on the

336 usefulness of APR scores from the viewpoint of proinflammatory cytokines. Dis Markers

337 2015:981981 DOI: 10.1155/2015/981981

338 Oyelese Y, Culin A, Ananth CV, Kaminsky LM, Vintzileos A, and Smulian JC. 2006.

339 Meconium-stained amniotic fluid across gestation and neonatal acid-base status. Obstet

340 Gynecol 108:345-349 DOI: 10.1097/01.AOG.0000226853.85609.8d

341 Ramin SM, Gilstrap LC, 3rd, Leveno KJ, Dax JS, and Little BB. 1993. Acid-base

342 significance of meconium discovered prior to labor. Am J Perinatol 10:143-145 DOI:

$34310.1055 / \mathrm{s}-2007-994647$

344 Redline RW, Faye-Petersen O, Heller D, Qureshi F, Savell V, Vogler C, and Society for

345 Pediatric Pathology PSAFINC. 2003. Amniotic infection syndrome: nosology and

346 reproducibility of placental reaction patterns. Pediatr Dev Pathol 6:435-448 DOI:

$347 \quad 10.1007 / \mathrm{s} 10024-003-7070-\mathrm{y}$

348 Romero R, Yoon BH, Chaemsaithong P, Cortez J, Park CW, Gonzalez R, Behnke E,

349 Hassan SS, Chaiworapongsa T, and Yeo L. 2014. Bacteria and endotoxin in meconium-

350 stained amniotic fluid at term: could intra-amniotic infection cause meconium passage? $J$

351 Matern Fetal Neonatal Med 27:775-788 DOI: 10.3109/14767058.2013.844124 
352 Speer C, Bruns A, and Gahr M. 1983. Sequential determination of CRP, alpha 1-

353 antitrypsin and haptoglobin in neonatal septicaemia. Acta Paediatr Scand 72:679-683.

354 Swarnam K, Soraisham AS, and Sivanandan S. 2012. Advances in the management of

355 meconium aspiration syndrome. Int J Pediatr 2012:359571 DOI: 10.1155/2012/359571

356 Thureen PJ, Hall DM, Hoffenberg A, and Tyson RW. 1997. Fatal meconium aspiration in

357 spite of appropriate perinatal airway management: pulmonary and placental evidence of

358 prenatal disease. Am J Obstet Gynecol 176:967-975.

359 Trimmer KJ, and Gilstrap LC, 3rd. 1991. "Meconiumcrit" and birth asphyxia. Am J

360 Obstet Gynecol 165:1010-1013.

361 van Ierland Y, and de Beaufort AJ. 2009. Why does meconium cause meconium

362 aspiration syndrome? Current concepts of MAS pathophysiology. Early Hum Dev

363 85:617-620 DOI: 10.1016/j.earlhumdev.2009.09.009

364 Yoder BA, Kirsch EA, Barth WH, and Gordon MC. 2002. Changing obstetric practices

365 associated with decreasing incidence of meconium aspiration syndrome. Obstet Gynecol $366 \quad 99: 731-739$.

367 Yoon BH, Romero R, Park JS, Kim M, Oh SY, Kim CJ, and Jun JK. 2000a. The 368 relationship among inflammatory lesions of the umbilical cord (funisitis), umbilical 369 cord plasma interleukin 6 concentration, amniotic fluid infection, and neonatal sepsis. 370 Am JObs

371 Yoon BH, Romero R, Park JS, Kim CJ, Kim SH, Choi JH, and Han TR. 2000b. Fetal exposure to 372 an intra-amniotic inflammation and the development of cerebral

373 palsy at the age of three years. Am J Obstet Gynecol 182:675-681. 
Figure 1

Profile of the study population

Ninety-five neonates, who were born through meconium-stained amniotic fluid and admitted to the neonatal intensive care unit within 24 hours of birth, were studied.

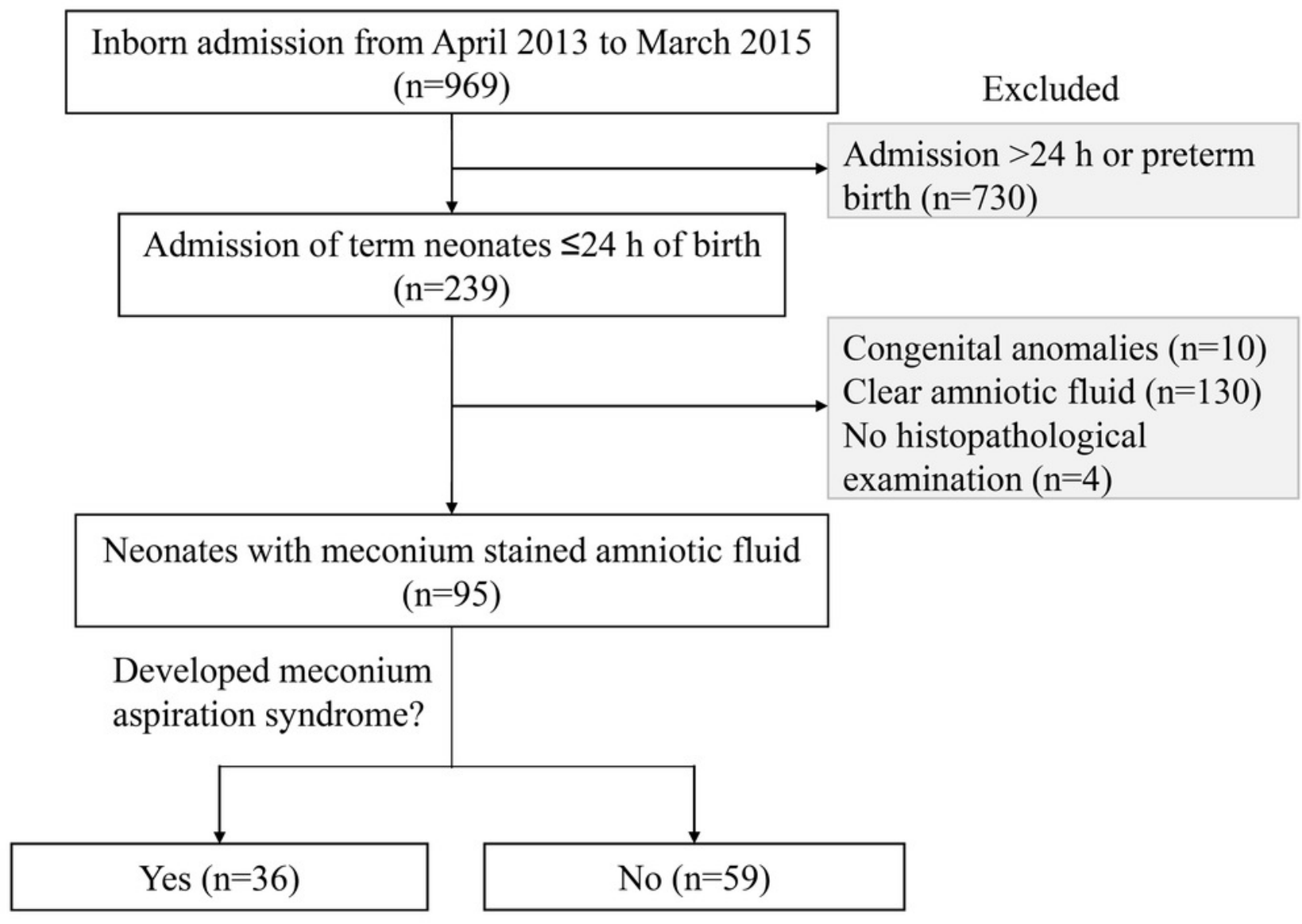




\section{Table 1 (on next page)}

Table $\square$ Independent variables of meconium aspiration syndrome development 


\section{Table}

2

3 Table 1: Independent variables of meconium aspiration syndrome development

\begin{tabular}{|c|c|c|c|c|c|c|}
\hline & \multirow{2}{*}{\multicolumn{2}{|c|}{ Meconium aspiration syndrome }} & \multicolumn{3}{|c|}{ Odds ratio } & \multirow{3}{*}{$p$-value } \\
\hline & & & \multirow{2}{*}{ Mean } & \multicolumn{2}{|c|}{$95 \% \mathrm{Cl}$} & \\
\hline & No $(n=59)$ & Yes $(n=36)$ & & Lower & Upper & \\
\hline \multicolumn{7}{|l|}{ Univariate model } \\
\hline Gestation (weeks) & $40.3 \pm 0.3$ & $40.5 \pm 0.4$ & 1.11 & 0.74 & 1.66 & 0.610 \\
\hline Birth weight (kg) & $3.02 \pm 0.56$ & $3.24 \pm 0.45$ & 2.38 & 1.00 & 5.66 & 0.050 \\
\hline Birth weight z-score & $-0.32 \pm 1.48$ & $0.21 \pm 1.25$ & 1.32 & 0.97 & 1.81 & 0.080 \\
\hline SGA $<2 S D$ of norm & 11(19) & $2(6)$ & 0.26 & 0.03 & 1.31 & 0.259 \\
\hline Male sex & $31(54)$ & $28(78)$ & 3.16 & 1.24 & 8.07 & 0.016 \\
\hline Delivery mode & & & 1.45 & 0.89 & 2.34 & 0.136 \\
\hline Vaginal & $34(58)$ & $16(44)$ & 0.56 & 0.24 & 1.29 & 0.171 \\
\hline Forceps/vacuum & $11(19)$ & $7(19)$ & 0.91 & 0.32 & 2.58 & 0.865 \\
\hline Caesarean & $12(20)$ & $13(36)$ & 2.15 & 0.87 & 5.33 & 0.097 \\
\hline PROM>24h & $13(22)$ & $11(31)$ & 1.56 & 0.61 & 3.98 & 0.355 \\
\hline 1-minute Apgar $\leq 7$ & $20(34)$ & $21(58)$ & 2.44 & 1.04 & 5.70 & 0.040 \\
\hline 5-minute Apgar $\leq 7$ & $12(20)$ & $9(25)$ & 1.12 & 0.41 & 3.07 & 0.827 \\
\hline Cord blood $\mathrm{pH}<7.1$ & $7(12)$ & $5(14)$ & 1.20 & 0.35 & 4.10 & 0.773 \\
\hline Cord blood $\mathrm{pH}<7.2$ & $22(37)$ & $19(53)$ & 1.88 & 0.81 & 4.36 & 0.141 \\
\hline Chorioamnionitis & $26(44)$ & $20(56)$ & 1.59 & 0.69 & 3.65 & 0.278 \\
\hline Funisitis & $35(59)$ & $30(83)$ & 3.43 & 1.24 & 9.50 & 0.018 \\
\hline \multicolumn{7}{|c|}{ Inflammatory biomarkers at admission } \\
\hline $\mathrm{CRP}(>0.3 \mathrm{mg} / \mathrm{dl})$ & $8(14)$ & $9(25)$ & 1.18 & 0.83 & 1.69 & 0.354 \\
\hline$\alpha 1-A G(>20 \mathrm{mg} / \mathrm{dl})$ & $38(64)$ & $28(78)$ & 1.01 & 1.00 & 1.03 & 0.101 \\
\hline $\mathrm{HP}(>13 \mathrm{mg} / \mathrm{dl})$ & $8(14)$ & $10(28)$ & 1.02 & 1.00 & 1.04 & 0.060 \\
\hline
\end{tabular}

Acute-phase inflammatory reaction score

\begin{tabular}{lcccccc}
0 & $21(36)$ & $8(22)$ & \multicolumn{5}{c}{ Reference } \\
1 or 2 & $33(55)$ & $19(53)$ & 1.59 & 0.59 & 4.26 & 0.356 \\
3 & $5(9)$ & $9(25)$ & 4.72 & 1.21 & 18.5 & 0.026 \\
\hline Multivariate model & & & & & \\
Funisitis & & 5.03 & 1.63 & 15.5 & 0.005 \\
1-minute Apgar $\leq 7$ & & 2.74 & 1.06 & 7.09 & 0.038 \\
Male sex & & 3.4 & 1.24 & 9.34 & 0.018
\end{tabular}

4 Notes: Values are shown as mean \pm standard deviation or number (\%). 
5 Abbreviations: CI, confidence interval. CRP, C-reactive protein. $\alpha_{1} \mathrm{AG}, \alpha_{1}$-acid glycoprotein. HP, 6 haptoglobin. SD, standard deviation. SGA, small for gestational age. 